\title{
Association between fatty liver disease markers and physical activity and sedentary time in children and adolescents with obesity
}

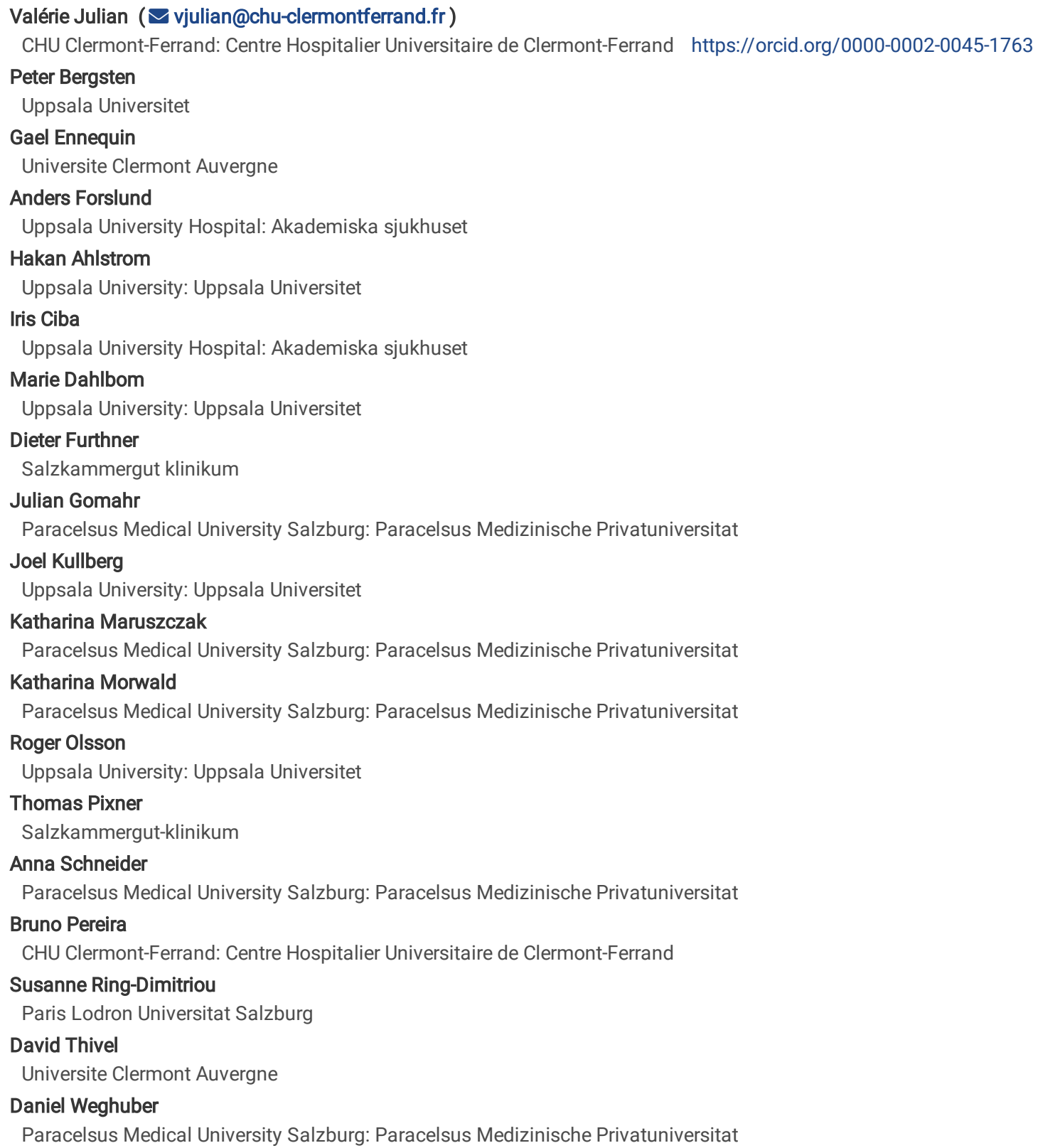

\section{Research Article}

Keywords: Sedentary behavior, Physical activity, Pediatric obesity, Nonalcoholic fatty liver disease

Posted Date: February 24th, 2022

DOI: https://doi.org/10.21203/rs.3.rs-1365849/v1

License: (c) (7) This work is licensed under a Creative Commons Attribution 4.0 International License. Read Full License 


\section{Abstract}

Purpose: To compare patterns of sedentary (SED) time (more sedentary, SED+ vs less sedentary, SED-), moderate to vigorous physical activity (MVPA) time (more active, MVPA+ vs less active, MVPA-) and combinations of behaviors (SED-/MVPA+, SED-/MVPA-, SED+/MVPA+, SED+/MVPA-) regarding nonalcoholic fatty liver diseases (NAFLD) markers.

Methods: This cross-sectional study included 134 subjects (13.4 $\pm 2.2 \mathrm{yrs}$, body mass index (BMI) $98.9 \pm 0.7$ percentile, $48.5 \%$ females) who underwent 24h/7day accelerometry, anthropometric and biochemical markers (alanine aminotransferase (ALT), aspartate aminotransferase (AST), gamma-glutamyl transpeptidase (GGT), AST/ALT and Fatty Liver Index (FLI)). A subgroup of 39 patients underwent magnetic resonance imaging-liver fat content (MRI-LFC).

Results: Hepatic health was better in SED- [lower ALT, GGT and MRI-LFC $(\mathrm{p}<0.05)$, higher AST/ALT $(\mathrm{p}<0.01)]$ vs SED+ and in MVPA+ [lower ALT ( $<<0.05)$ and FLI ( $p=0.001)$, higher AST/ALT $(p<0.01)]$ vs MVPA- groups after adjustment for age, gender and Tanner stages. SED-/MVPA+ group had the best hepatic health. SED-/MVPA- group had lower ALT and GGT and higher AST/ALT $(p<0.05)$ in comparison with SED+/MVPA+ group independently of BMI. SED time was positively associated with biochemical (high ALT, low AST/ALT ratio) and imaging (high MRI-LFC) markers independently of MVPA. MVPA time was associated with biochemical markers (low ALT, FLI, high AST/ALT) but these associations were no longer significant after adjustment for SED time, except for FLI $(p<0.001)$

Conclusions: Lower SED time is associated with better hepatic health independently of MVPA. Reducing SED time might be a first step in the management of pediatric obesity NAFLD when increasing MVPA is not possible.

\section{What Is Known}

- MVPA and SED times are associated with cardiometabolic risks in youths with obesity

- The relationships between NAFLD markers and concomittant MVPA and SED times have not been studied in this population

\section{What Is New}

- Low SED time is associated with healthier liver enzymes profiles and LFC independent of MVPA

- While low SED/high MVPA is the more desirable pattern, low SED/low MVPA pattern would have healthier liver enzymes profile compared with high MVPA/high SED, independent of BMI, suggesting that reducing SED time irrespective of MVPA is needed to optimize liver health

\section{Introduction}

Pediatric obesity is associated with cardiometabolic diseases (insulin resistance, type 2 diabetes, dyslipidemia, high blood pressure) and nonalcoholic fatty liver diseases (NAFLD) [1, 2]. Defined as an excess of hepatic fat, NAFLD has become the most common liver disease in childhood, regarded as a hepatic manifestation of the metabolic syndrome (MetS) [3]. Youths with obesity and MetS have 5 times the odds of having NAFLD compared with their counterparts with obesity alone, and youths with both obesity and NAFLD have higher insulin resistance, dyslipidemia and blood pressure than youths with obesity alone [4]. As obesity and related cardiovascular and metabolic diseases track into adulthood, there is a need of a life course perspective in pediatric obesity prevention and treatment. Multidisciplinary interventions combining nutritional guidelines and physical activity (PA), particularly moderate to vigorous PA (MVPA), remain the cornerstone therapy of pediatric obesity and related-diseases [3, 5-7]. Behavioral recommendations, that mainly focused on MVPA so far, also advocate now for the minimization of sedentary (SED) time (i.e. waking behavior in a sitting, reclining or lying posture), synergistically with MVPA increase [8]. Both high MVPA and low SED times have been associated with healthier profiles of liver enzymes and reduced liver fat content (LFC) in adults [913]. In youths, while evidence supports that increasing MVPA and decreasing SED time reduce cardiometabolic risks [14-17], relationships between accelerometry-based PA levels and NAFLD markers remain understudied [18-20], and only few studies investigated so far the association between SED time and NAFLD surrogate markers (without imaging), showing discrepant results $[18,19,21]$. To our knowledge, no study have measured concomitantly PA and SED times in regard to NAFLD biomarkers and LFC in youths with obesity.

As it remains difficult to implement MVPA in youths with obesity in clinical practice, our team conducted a cross sectional study comparing 7day/24h accelerometry-based patterns of SED (more sedentary, SED + vs less sedentary, SED -), MVPA (more active, MVPA + vs less active, MVPA-) and combinations of SED and MVPA times (SED-/MVPA+, SED-/MVPA-, SED+/MVPA+, SED+/MVPA-). Results showed that i) low SED/high MVPA pattern had the most desirable cardiometabolic profile, ii) SED-/MVPA- pattern had lower fat mass, insulin resistance, dyslipidemia and metabolic syndrome risk score, independent of BMI, in comparison with SED+/MVPA + pattern, and iii) SED time was correlated with cardiometabolic risks independently of MVPA [22, 23]. Considering the strong relationships between cardiometabolic comorbidities and NAFLD [4, 24], it seemed relevant to question whether movement behaviors patterns might be associated with NAFLD markers in the same population.

Therefore, the present study aimed to i) compare patterns of SED, MVPA and combinations of SED and MVPA times regarding liver enzymes (alanine aminotransferase, ALT, as primary criterion) and LFC and ii) investigate correlations between SED time, PA levels and NAFLD markers in children and adolescents with obesity.

\section{Materials And Methods}

\section{Participants}


This cross-sectional study involved 134 children and adolescents with obesity participating in the betaJUDO study[25]. They were included in 2 centers (Pediatric Obesity Clinic at University Children's Hospital, Uppsala, Sweden, and Paracelsus Medical University, Salzburg, Austria). Inclusion criteria were the followings: (a) 10-17 years old, (b) age-adapted BMI > 30kg. $\mathrm{m}^{-2}$, (c) medical examination including anthropometric assessment and Tanner's staging, (d) at least 5 valid days out of a possible 7 days of accelerometry measurements, including one of the valid day on a weekend (regardless of PA and SED levels), (e) blood samplings for liver parameters, ( $f$ ) no contraindication to PA, (g) no additional medical/psychiatric conditions nor medication influencing cardiometabolic, liver or accelerometry data. The study was accepted for Voluntary Harmonisation Procedure (VHP673, VHP2015061) and approved by Ethics Committees and Regulatory Authorities (EudraCT No: 2015-001628-45; EC Sweden: Dnr 2015/279; EC Austria: 415-E/1544/20-2014). Written informed consent were obtained from participants and parents. The trial was conducted according to the Declaration of Helsinki (World Medical Association; Version 2013) and the E6 Guideline for Good Clinical Practice (International Conference on Harmonisation).

\section{Anthropometry and pubertal staging}

Standard operating procedures for measurements were harmonized between centers [25]. Weight $(\mathrm{kg})$ was assessed using a standardized calibrated scale (Uppsala: SECA model 704; Salzburg: SECA model 801, Hamburg, Germany). Height (cm) was measured using a stadiometer (Uppsala: Ulmer stadiometer, Busse, Elchingen, Germany; Salzburg: SECA, model 222 stadiometer, Hamburg, Germany). BMI was calculated as weight (kilograms) divided by the square of height (meters). BMI-SDS (Microsoft Excel add-in LMS Growth using WHO growth report Version 2.76) and BMI percentiles (WHO BMI for age) were calculated. Waist circumference $(\mathrm{WC}, \mathrm{cm})$ was measured with a flexible tape midway between the superior border of the iliac crest and the lowest rib on a standing patient. Fat mass (FM) percentage was calculated using an InBody S20 bioimpedance device (Biospace, Seoul, Korea) at fasting. Puberty was evaluated with Tanner staging $[26,27]$.

\section{Biochemical variables}

Blood was sampled at fasting. Validation of analyses was performed between laboratories [25]. Serum concentrations of liver enzymes (ALT, aspartate aminotransferase (AST) and gamma-glutamyl transpeptidase (GGT)), total-cholesterol, high density lipoprotein-cholesterol (HDL-c), low density lipoproteincholesterol (LDL-c) and triglycerides (TG) were analyzed by enzymatic photometric analysis. Plasma glucose was analyzed by enzymatic chromatic test. Plasma was used for central analyses of insulin using singleplex enzyme-linked immunosorbent assay kits for each analyte (Mercodia AB, Uppsala, Sweden). Insulin resistance was expressed using homeostasis model assessment of insulin-resistance index (HOMA-IR), [HOMA-IR] $=$ glycemia $\left[\mathrm{mmol} \times \mathrm{L}^{-1}\right] \times$ insulinemia $\left.\left[\mathrm{mUl} \times \mathrm{L}^{-1}\right] / 22.5\right)[28]$.

\section{Fatty liver index calculation}

Fatty Liver Index (FLI) was calculated as followed:

$F L I=e^{f} /\left(1+e^{f}\right) \times 100$

$f=0.953 \times \ln$ (triglycerides) $+0.139 \times B M I+0.718 \times \ln (g g t)+$

$0.053 \times W C-15.745)$

(TG in $\mathrm{mg} / \mathrm{dL}, \mathrm{GGT}$ in $\mathrm{UI} / \mathrm{L}$, WC in $\mathrm{cm}$ )

Although initially developed for adults, FLI is currently used in pediatric clinical trials as a biomarker of steatosis and varies between 0 and 100 [29-31].

\section{Percentage hepatic fat}

Percentage hepatic fat was measured by magnetic resonance imaging (MRI) using 1.5 Tesla clinical MRI systems from Philips Medical System (Best, The Netherlands; Uppsala: Philips Achieva system, Salzburg: Philips Ingenia system) [25]. Children were categorized as having hepatic steatosis when percentage hepatic fat $\geq 5 \%$ [32].

\section{Physical activity and sedentary time}

Movements related behaviors were assessed with the accelerometer Actical ${ }^{\circledR}$ (Philips Respironics, Inc, Murrysville, PA). As previously described [23], it is an omni-directional waterproof device recording accelerations in the range of $0.05-2.0 \mathrm{~g}$, sensitive to movements in the range of $0.35-3.5 \mathrm{~Hz}$, and able to record the magnitude of acceleration and deceleration associated with every movement. The signal was scored as a "count" which was summed over a 1 minute epoch. Participants were asked to wear the device on their non-dominant wrist during 7 consecutive days (24-hours measurements). Non-wear time was defined as $\geq 60$ consecutive minutes of zero counts, with allowance for 2 min of counts between zero and 100 . Wear time was determined by subtracting nonwear time from 24 hours. A valid day was defined as $\geq 10 \mathrm{~h}$ of wear time. Each minute of wear time was classified using established cut points into SED ( $<1.5$ metabolic equivalent of the task, METS), light physical activity (LPA, $<3$ METS), moderate activity (MPA, 3 to 6 METS) and vigorous activity (VPA, $>6$ METS) [33]. MVPA was the sum of MPA and VPA. SED+ (more sedentary) and SED- (less sedentary) groups were defined by being respectively upper and under the median of the sample for SB time. MVPA+ (more active) and MVPA- (less active) groups were defined by being respectively upper and under the median of the sample for MVPA time. The 4 combinations (SED-/MVPA+, SED-/MVPA-, SED+/MVPA+, SED+/MVPA-) were created using the median of MVPA for each of SED subsamples.

\section{Statistical Analysis}


Statistical analyses were performed using Stata software (version 15, StataCorp, College Station, US). Continuous data were expressed as means and standard-deviations (SD). The normality of the distribution was checked with a Shapiro-Wilk test. Comparisons between groups were performed using Chisquared or Fisher's exact test for categorical data, and analysis of variance (ANOVA) or non-parametric Kruskal-Wallis test (when the ANOVA assumptions were not met) for continuous variables. Assumption of homoscedasticity was studied using Bartlett's test. Whan appropriate (omnibus p-value<0.05), post-hoc test for two by two multiple comparison were applied: Tukey-Kramer after ANOVA, Dunn after Kruskal-Wallis test and Marascuilo for categorical data. Relationships between continuous data were explored with Pearson or Spearman correlation coefficient and a Sidak type I error correction. Multivariable analyses were conducted using multiple linear regression in order to compare groups adjusting aforementioned analyses on possible confounders. No specific strategy approach, such as stepwise, was conducted. Covariates were chosen according to univariate results and clinical relevance. Multivariable regression analyses were run with the following covariates: age, gender and Tanner stages (model 2) and age, gender, Tanner stages and BMI (model 3). The normality of residuals was checked and a logarithmic transformation of the dependent variable was performed when appropriate. Differences were considered statistically significant at $p<0.05$.

\section{Results}

One hundred and thirty-four adolescents (mean age $13.4 \pm 2.2 \mathrm{yrs}, 48.5 \%$ females) were included ( $\mathrm{n}=97$ from Uppsala and $\mathrm{n}=37$ from Salzburg). The mean BMI was $98.9 \pm 0.7 \mathrm{~kg} \cdot \mathrm{m}^{-2}$. Mean accelerometry wear time was $6.5 \pm 1.1$ days with $99.4 \pm 2.9$ percent of daily wear time. All the patients underwent anthropometric measurements, accelerometry and biochemical assessments. A subgroup of thirty-nine patients underwent MRI-LFC measurements. Anthropometrics, accelerometry and hepatic variables for overall sample, SED- and SED+ groups, MVPA- and MVPA+ groups, are presented in Table 1.

\section{Comparison SED+ vs SED- groups and MVPA- vs MVPA+ groups}

In univariate analysis (model 1), SED+ group had higher ALT ( $p=0.016)$, GGT (0.019), FLI ( $p=0.004)$ and MRI-LFC ( $p=0.012)$, with a lower AST/ALT ratio $(p<0.001)$ in comparison with SED- group. These results remained significant when adjusted for age, gender and Tanner stages (model 2$)$, except for FLI $(p=0.131)$, and when adjusted for age, gender, Tanner stages and BMI (model 3), except for GGT ( $p=0.055)$. For the three models, the number of subjects with a MRI-LFC $\geq 5 \%$ tend to be higher in the SED + group in comparison to SED- group, without however reaching significance ( $p=0.064, p=0.056$, and $p=0.067$, respectively) (Table 1$)$.

In univariate analysis (model 1), MVPA- group had higher ALT ( $p=0.003)$, GGT $(p=0.049)$ and FLI $(p<0.001)$, with a lower AST/ALT ratio ( $p<0.001)$ in comparison with MVPA+ group. These results remained significant when adjusted for age, gender and Tanner stages (model 2) and when adjusted for age, gender, Tanner stages and BMI (model 3), except for GGT ( $p=0.298$ and $p=0.363$, respectively) (Table 1).

Results regarding insulin resistance and lipid profile have been published elsewhere [22] and are presented in supplementary files for overall sample, SED- and SED+ groups, MVPA- and MVPA+ groups (Supplementary Table 1).

\section{Comparison between SED-/MVPA+, SED-/MVPA-, SED+/ MVPA+ and SED+/MVPA- groups}

Anthropometrics, accelerometry and hepatic variables for SED-/MVPA+, SED-/MVPA-, SED+/MVPA+ and SED+/MVPA- groups are presented in Table 2 .

SED-/MVPA+ group had a lower MRI-LFC $(p<0.01)$ in comparison with the SED+/MVPA- group, and lower ALT $(p<0.01)$ and GGT $(p<0.05)$ with a higher AST/ALT ratio $(p<0.01)$ in comparison with the SED+/MVPA+ and SED+/MVPA- groups, after adjustment with age, gender and Tanner stages.

SED-/MVPA- group had lower SED time $(p<0.001)$ but lower MVPA time $(p<0.001)$ and higher LPA time $(p<0.001)$ in comparison with SED+/MVPA+ group. BMI was not different between the 2 groups $(p=0.108)$. However, after adjustment with age, gender and Tanner stages, SED-/MVPA- group had lower ALT ( $<0.05$ ) and GGT $(p<0.05)$ and higher AST/ALT ratio $(p<0.05)$, in comparison with SED+/MVPA+ group (Table 2). These results remained significant after adjustment with age, gender, Tanner stages and BMI $(\mathrm{p}<0.05)$.

Results regarding insulin resistance and lipid profile have been published elsewhere [22] and are presented in supplementary files for SED-/MVPA+, SED-/MVPA-, SED+/ MVPA+ and SED+/MVPA-groups (Supplementary Table 2).

\section{Correlations}

All correlations between hepatic variables and accelerometry variables are presented in Fig. 1. SED time was positively correlated with ALT ( $\mathrm{p}<0.05$ ), FLI $(p<0.05)$ and MRI-LFC $(p<0.01)$, and negatively correlated with AST/ALT ratio $(p<0.001)$. SED time remained positively correlated with ALT ( $<<0.05)$ and MRILFC $(p<0.05)$ and negatively correlated with AST/ALT ratio $(p<0.05)$ after adjustment with MVPA time. These associations remained significant after adjustment for age, gender, Tanner stages, BMI and MVPA time $(\mathrm{p}<0.05)$.

MVPA was negatively correlated with ALT $(p<0.05)$ and FLI $(p<0.001)$, and positively correlated with AST/ALT ratio ( $p<0.001)$. MVPA tend to be associated with MRI-LFC, without however reaching significance $(p=0.056)$. MVPA remained negatively correlated with FLI $(p<0.001)$ after adjustment with SED time.

\section{Discussion}

This study aimed to investigate the association between SED and MVPA patterns measured by 24h/7 days accelerometry and NAFLD markers in children and adolescents with obesity. According to our analysis, although SED-/MVPA + pattern is associated with the best hepatic health surrogates, juveniles with SED-/MVPA- pattern had better biochemical hepatic markers in comparison with those with SED+/MVPA + pattern, independently of BMI. Moreover, SED time was positively associated with biochemical (high ALT, low AST/ALT ratio) and imaging (high MRI-LFC) markers of hepatic health independently of MVPA.

Page $4 / 11$ 
MVPA time was associated with biochemical markers of hepatic health (low ALT, FLI, high AST/ALT ratio), but these associations were no longer significant after adjustment for SED time (except for FLI). To our knowledge, this is the first study to measure objectively and concomitantly PA and SED times in regard to NAFLD biomarkers and MRI-LFC in youths with obesity. The results demonstrate the importance of a reduced SED time on hepatic health, irrespectively of MVPA, in youths with obesity.

In the subgroup who underwent liver MRI, the proportion of subjects having a LFC $\geq 5 \%$ was $46 \%(n=18 / 39)$, which is in keeping with previous studies showing a large variability in NAFLD prevalence in youths with obesity, depending on the diagnostic method [7, 20, 34-36]. In a meta-analysis based on various diagnostic methods (ALT, ultrasonography and MRI), NAFLD prevalence was estimated at $34 \%$ in a large sample of 23892 youths under 14 years old [34]. Studies measuring percentage hepatic fat by MRI estimated a prevalence between 30 and $44 \%$ depending on the cut-off points of hepatic fat fraction [20, $35,36]$.

The better hepatic health in SED-/MVPA + group is fully in line with the more favorable cardiometabolic health found in youths meeting guidelines for both MVPA and SED times $[22,37,38]$. The present results strengthens a body of evidence promoting PA recommendations in order to optimize liver health in young subjects [18-20,34,39]. Moreover, the comparison between SED-/MVPA- and SED+/MVPA + groups and the mutually adjusted model of correlations between movements-related behaviors and NAFLD markers strongly highlights the importance of SED time in determining optimal liver health, independent of MVPA level. This is concordant with previous findings in adults reporting a positive independent association between SED time and LFC [10, 12, 13]. Interestingly, Li et al recently showed in more than 16000 adults that after adjusting for MVPA, increasing quartiles of SED time were associated with a higher prevalence of elevated ALT and GGT, which remained significant after further adjustment for cardiometabolic traits (including BMI, lipids and HOMA-IR) [9]. In contrast, increasing quartiles of MVPA were associated with a lower prevalence of elevated ALT after adjustment for SED time, but this became non-significant after further adjustment for cardiometabolic traits [9]. In youths, while evidence now supports that decreasing SED time reduces cardiometabolic risks [22, 4042], only Martins et al previously found a positive association between SED time and ALT [19]. Norman et al however showed that screen time, a main contributor to SED time, was positively associated with cardiometabolic parameters and ALT, independent of MVPA time, in adolescents with obesity [21], suggesting that limiting screen time would represent an independent lever of action to improve hepatic health. All these results are in line with recently proposed anti-obesity strategies aiming at breaking up prolonged periods of SED time in youths, replacing it with MVPA (the preferred and more efficient scenario) or with LPA (beneficial but with lower effect estimates) [43, 43-45]. Winters-van Eekelen recently showed that reallocation of SED time with MVPA was associated with less total, visceral and liver fat in adults [46].

The present results have to be considered in light of some limitations. First, only a subgroup of subjects underwent liver MRI. Although MRI-LFC has been found correlated with SED time, the absence of correlations between MRI-LFC and MVPA might be explained by the small sample size. However, although liver enzymes alone are inadequate for identification of hepatic steatosis in children, ALT remains the best biochemical marker of hepatocellular lipid content [47]. It has been associated with insulin resistance and cardiometabolic health in adolescents [48]. Secondly, as previously discussed [22, 23], while accelerometry is the gold standard method for measuring movements-related behaviors, MVPA has been potentially overestimated at the expense of LPA. While Actical was shown comparable to the Actigraph GT3X in youths [49], Migueles et al. recently demonstrated that MVPA time largely differed across attachment sites (hip vs wrist), acceleration metrics (number of days and hours per day) and cut-points in youths with obesity [50]. These parameters were however similar for all the participants of the present study. The wrist attachment site had been chosen to increase wear compliance [26], which is a strength of the present study, providing measurements with more than $99 \%$ of wear time during a mean of 6.5 days. Finally, Swedish children are known to have a meaningful $50 \%$ higher MVPA than children of other European countries [51].

While recommendations have largely focused on MVPA so far, the present study reinforces the need to reduce SED time, synergistically with the increase in MVPA time, to optimize liver health in youths with obesity. In clinical practice, youths with obesity should benefit from an individual behavioral diagnostic targeting all movements behaviors, and stakeholders should be sensitized to support the shift from long periods of SED time to daily routines incorporating bouts of PA. Furthermore, reducing SED time might be a first step in the management of pediatric obesity NAFLD when increasing MVPA is not possible. Longterm cohort studies on the impact of changes in PA and SED times on hepatic health outcomes, and interventional studies with a sequential action plan (i.e. sequencing the actions into a first phase with the aim to reduce SED time followed by a classic supervised MVPA training), are needed.

\section{Abbreviations}

ALT: Alanine aminotransferase; AST: Aspartate aminotransferase; BMI: Body mass index; FLI: Fatty liver index; GGT: gamma-glutamyl transpeptidase; HDL-C: High density lipoprotein cholesterol; HOMA-IR: Homeostasis model assessment of insulin-resistance; LDL-c: low density lipoprotein cholesterol; LFC: Liver fat content; LPA: Low physical activity; METS: Metabolic equivalent of the task; MPA: Moderate physical activity; MRI: Magnetic resonance imaging; MVPA: Moderate to vigorous physical activity; NAFLD: Nonalcoholic fatty liver disease; PA: Physical activity; SED: Sedentary; TG: triglycerides; VPA: Vigorous physical activity; WC: Waist circumference

\section{Declarations}

Fundings: This research was funded by European Union Seventh Framework Programme (FP7/2007-2013) under grant agreement number 279153 (BetaJUDO). In addition, support for the study was received from the Regional Research Council in Uppsala-Örebro, Sweden, the Swedish Diabetes Foundation, the Swedish Society for Diabetology and the Swedish Research Council (2016-01040).

Conflicts of Interest/Competing Interests: The authors have no relevant financial or non-financial interests to disclose.

Availability of data and material: not available 
Code availability: not applicable

Authors contributions: All authors contributed to the study conception and design. Material preparation, data collection and analysis were performed by Peter Bergsten, Anders Forslund, Hakan Ahlstrom, Iris Ciba, Marie Dahlbom, Dieter Furthner, Julian Gomahr, Joel Kullberg, Katharina Maruszczak, Katharina Morwald, Roger Olsson, Thomas Pixner, Anna Schneider, Bruno Pereira and Susanne Ring-Dimitriou. The first draft of the manuscript was written by Valérie Julian, David Thivel and Daniel Weghuber and all authors commented on previous versions of the manuscript. All authors read and approved the final manuscript.

Compliance with Ethical Standards: The study was accepted for Voluntary Harmonisation Procedure (VHP673, VHP2015061) and approved by Ethics Committees and Regulatory Authorities (EudraCT No: 2015-001628-45; EC Sweden: Dnr 2015/279; EC Austria: 415-E/1544/20-2014). The trial was conducted according to the Declaration of Helsinki (World Medical Association; Version 2013) and the E6 Guideline for Good Clinical Practice (International Conference on Harmonisation).

Consent to participate: Written informed consent were obtained from participants and parents.

Consent for publication: not applicable

\section{References}

1. Kansra AR, Lakkunarajah S, Jay MS (2021) Childhood and Adolescent Obesity: A Review. Front Pediatr 8. https://doi.org/10.3389/fped.2020.581461

2. Shapiro WL, Noon SL, Schwimmer JB (2021) Recent advances in the epidemiology of nonalcoholic fatty liver disease in children. Pediatr Obes e12849. https://doi.org/10.1111/ijpo.12849

3. Temple JL, Cordero P, Li J et al (2016) A Guide to Non-Alcoholic Fatty Liver Disease in Childhood and Adolescence. Int J Mol Sci 17:E947. https://doi.org/10.3390/ijms17060947

4. Schwimmer JB, Pardee PE, Lavine JE et al (2008) Cardiovascular risk factors and the metabolic syndrome in pediatric nonalcoholic fatty liver disease. Circulation 118:277-283. https://doi.org/10.1161/CIRCULATIONAHA.107.739920

5. Boff R, de Liboni M, de Batista RPA et al (2017) Weight loss interventions for overweight and obese adolescents: a systematic review. Eat Weight Disord 22:211-229. https://doi.org/10.1007/s40519-016-0309-1

6. Albert Pérez E, Mateu Olivares V, Martínez-Espinosa RM et al (2018) New Insights about How to Make an Intervention in Children and Adolescents with Metabolic Syndrome: Diet, Exercise vs. Changes in Body Composition. A Systematic Review of RCT. Nutrients 10:E878. https://doi.org/10.3390/nu10070878

7. Vos MB, Abrams SH, Barlow SE et al (2017) NASPGHAN Clinical Practice Guideline for the Diagnosis and Treatment of Nonalcoholic Fatty Liver Disease in Children: Recommendations from the Expert Committee on NAFLD (ECON) and the North American Society of Pediatric Gastroenterology, Hepatology and Nutrition (NASPGHAN). J Pediatr Gastroenterol Nutr 64:319-334. https://doi.org/10.1097/MPG.0000000000001482

8. Chaput J-P, Willumsen J, Bull F et al (2020) 2020 WHO guidelines on physical activity and sedentary behaviour for children and adolescents aged 5-17 years: summary of the evidence. Int J Behav Nutr Phys Act 17:141. https://doi.org/10.1186/s12966-020-01037-z

9. Li J, Hua S, Chen G-C et al (2020) Objectively measured sedentary time, physical activity and liver enzyme elevations in US Hispanics/Latinos. Liver Int 40:1883-1894. https://doi.org/10.1111/liv.14514

10. Bowden Davies KA, Sprung VS, Norman JA et al (2019) Physical Activity and Sedentary Time: Association with Metabolic Health and Liver Fat. Med Sci Sports Exerc 51:1169-1177. https://doi.org/10.1249/MSS.0000000000001901

11. Long MT, Pedley A, Massaro JM et al (2015) Hepatic steatosis is associated with lower levels of physical activity measured via accelerometry. Obes (Silver Spring) 23:1259-1266. https://doi.org/10.1002/oby.21058

12. Henson J, Edwardson CL, Morgan B et al (2015) Associations of Sedentary Time with Fat Distribution in a High-Risk Population. Med Sci Sports Exerc 47:1727-1734. https://doi.org/10.1249/MSS.0000000000000572

13. Henson J, Edwardson CL, Morgan B et al (2018) Sedentary Time and MRI-Derived Measures of Adiposity in Active Versus Inactive Individuals. Obesity 26:29-36. https://doi.org/10.1002/oby.22034

14. Janssen I, Leblanc AG (2010) Systematic review of the health benefits of physical activity and fitness in school-aged children and youth. Int J Behav Nutr Phys Act 7:40. https://doi.org/10.1186/1479-5868-7-40

15. Poitras VJ, Gray CE, Borghese MM et al (2016) Systematic review of the relationships between objectively measured physical activity and health indicators in school-aged children and youth. Appl Physiol Nutr Metab 41:S197-239. https://doi.org/10.1139/apnm-2015-0663

16. Renninger M, Hansen BH, Steene-Johannessen $\mathrm{J}$ et al (2020) Associations between accelerometry measured physical activity and sedentary time and the metabolic syndrome: A meta-analysis of more than 6000 children and adolescents. Pediatr Obes 15:e12578. https://doi.org/10.1111/ijpo.12578

17. Carson V, Tremblay MS, Chaput J-P, Chastin SFM (2016) Associations between sleep duration, sedentary time, physical activity, and health indicators among Canadian children and youth using compositional analyses. Appl Physiol Nutr Metab 41:S294-302. https://doi.org/10.1139/apnm-2016-0026

18. Ruiz JR, Labayen I, Ortega FB et al (2014) Physical activity, sedentary time, and liver enzymes in adolescents: the HELENA study. Pediatr Res 75:798-802. https://doi.org/10.1038/pr.2014.26

19. Martins C, Aires L, Júnior IF et al (2015) Physical Activity is Related to Fatty Liver Marker in Obese Youth, Independently of Central Obesity or Cardiorespiratory Fitness. J Sports Sci Med 14:103-109

Page 6/11 
20. Medrano M, Arenaza L, Migueles JH et al (2020) Associations of physical activity and fitness with hepatic steatosis, liver enzymes, and insulin resistance in children with overweight/obesity. Pediatr Diabetes 21:565-574. https://doi.org/10.1111/pedi.13011

21. Norman GJ, Carlson JA, Patrick K et al (2017) Sedentary Behavior and Cardiometabolic Health Associations in Obese 11-13-Year Olds. Child Obes 13:425-432. https://doi.org/10.1089/chi.2017.0048

22. Julian V, Bergsten P, Forslund A et al (2022) Sedentary time has a stronger impact on metabolic health than moderate to vigorous physical activity in adolescents with obesity: a cross-sectional analysis of the Beta-JUDO study. https://doi.org/10.1111/ijpo.12897. Pediatric Obesity

23. Julian V, Ciba I, Olsson R et al (2021) Association between Metabolic Syndrome Diagnosis and the Physical Activity-Sedentary Profile of Adolescents with Obesity: A Complementary Analysis of the Beta-JUDO Study. Nutrients 14:60. https://doi.org/10.3390/nu14010060

24. Koutny F, Stein R, Kiess W et al (2021) Elevated transaminases potentiate the risk for emerging dysglycemia in children with overweight and obesity. Pediatr Obes 16:e12822. https://doi.org/10.1111/ijpo.12822

25. Forslund A, Staaf J, Kullberg J et al (2014) Uppsala Longitudinal Study of Childhood Obesity: Protocol Description. Pediatrics 133:e386-e393. https://doi.org/10.1542/peds.2013-2143

26. Rosenberger ME, Buman MP, Haskell WL et al (2016) Twenty-four Hours of Sleep, Sedentary Behavior, and Physical Activity with Nine Wearable Devices. Med Sci Sports Exerc 48:457-465. https://doi.org/10.1249/MSS.0000000000000778

27. Marshall WA, Tanner JM (1970) Variations in the pattern of pubertal changes in boys. Arch Dis Child 45:13-23. https://doi.org/10.1136/adc.45.239.13

28. Henderson M, Rabasa-Lhoret R, Bastard J-P et al (2011) Measuring insulin sensitivity in youth: How do the different indices compare with the goldstandard method? Diabetes Metab 37:72-78. https://doi.org/10.1016/j.diabet.2010.06.008

29. Bedogni G, Tamini S, Caroli D et al (2021) Development and Internal Validation of Fatty Liver Prediction Models in Obese Children and Adolescents. J Clin Med 10:1470. https://doi.org/10.3390/jcm10071470

30. Ferraioli G, Calcaterra V, Lissandrin R et al (2017) Noninvasive assessment of liver steatosis in children: the clinical value of controlled attenuation parameter. BMC Gastroenterol 17:61. https://doi.org/10.1186/s12876-017-0617-6

31. Koot BGP, van der Baan-Slootweg OH, Bohte AE et al (2013) Accuracy of prediction scores and novel biomarkers for predicting nonalcoholic fatty liver disease in obese children. Obes (Silver Spring) 21:583-590. https://doi.org/10.1002/oby.20173

32. Chalasani N, Younossi Z, Lavine JE et al (2012) The diagnosis and management of non-alcoholic fatty liver disease: Practice guideline by the American Association for the Study of Liver Diseases, American College of Gastroenterology, and the American Gastroenterological Association. Am J Gastroenterol 107:811-826. https://doi.org/10.1038/ajg.2012.128

33. Crouter SE, Bassett DR (2008) A new 2-regression model for the Actical accelerometer. Br J Sports Med 42:217-224. https://doi.org/10.1136/bjsm.2006.033399

34. Anderson EL, Fraser A, Howe LD et al (2016) Physical Activity Is Prospectively Associated With Adolescent Nonalcoholic Fatty Liver Disease. J Pediatr Gastroenterol Nutr 62:110-117. https://doi.org/10.1097/MPG.0000000000000904

35. Radetti G, Kleon W, Stuefer J, Pittschieler K (2006) Non-alcoholic fatty liver disease in obese children evaluated by magnetic resonance imaging. Acta Paediatr 95:833-837. https://doi.org/10.1080/08035250500449890

36. Kim G, Giannini C, Pierpont B et al (2013) Longitudinal effects of MRI-measured hepatic steatosis on biomarkers of glucose homeostasis and hepatic apoptosis in obese youth. Diabetes Care 36:130-136. https://doi.org/10.2337/dc12-0277

37. Roman-Viñas B, Chaput J-P, Katzmarzyk PT et al (2016) Proportion of children meeting recommendations for 24-hour movement guidelines and associations with adiposity in a 12-country study. Int J Behav Nutr Phys Act 13:123. https://doi.org/10.1186/s12966-016-0449-8

38. Saunders TJ, Gray CE, Poitras VJ et al (2016) Combinations of physical activity, sedentary behaviour and sleep: relationships with health indicators in school-aged children and youth. Appl Physiol Nutr Metab 41:S283-293. https://doi.org/10.1139/apnm-2015-0626

39. González-Ruíz K, Correa-Bautista JE, Izquierdo M et al (2021) Exercise dose on hepatic fat and cardiovascular health in adolescents with excess of adiposity. https://doi.org/10.1111/ijpo.12869. Pediatr Obes e12869

40. Jakubec L, Gába A, Dygrýn J et al (2020) Is adherence to the 24-hour movement guidelines associated with a reduced risk of adiposity among children and adolescents? BMC Public Health 20:1119. https://doi.org/10.1186/s12889-020-09213-3

41. Mann KD, Howe LD, Basterfield L et al (2017) Longitudinal study of the associations between change in sedentary behavior and change in adiposity during childhood and adolescence: Gateshead Millennium Study. Int J Obes (Lond) 41:1042-1047. https://doi.org/10.1038/ijo.2017.69

42. Schwarzfischer P, Gruszfeld D, Socha $P$ et al (2018) Longitudinal analysis of physical activity, sedentary behaviour and anthropometric measures from ages 6 to 11 years. Int J Behav Nutr Phys Act 15:126. https://doi.org/10.1186/s12966-018-0756-3

43. Gába A, Pedišić Ž, Štefelová N et al (2020) Sedentary behavior patterns and adiposity in children: a study based on compositional data analysis. BMC Pediatr 20:147. https://doi.org/10.1186/s12887-020-02036-6

44. Wijndaele K, White T, Andersen LB et al (2019) Substituting prolonged sedentary time and cardiovascular risk in children and youth: a meta-analysis within the International Children's Accelerometry database (ICAD). Int J Behav Nutr Phys Act 16:96. https://doi.org/10.1186/s12966-019-0858-6

45. Grgic J, Dumuid D, Bengoechea EG et al (2018) Health outcomes associated with reallocations of time between sleep, sedentary behaviour, and physical activity: a systematic scoping review of isotemporal substitution studies. Int J Behav Nutr Phys Activity 15:69. https://doi.org/10.1186/s12966-018-06913

46. Winters-VAN Eekelen E, Velde VANDER, Boone JHPM et al (2021) Objectively Measured Physical Activity and Body Fatness: Associations with Total Body Fat, Visceral Fat, and Liver Fat. Med Sci Sports Exerc 53:2309-2317. https://doi.org/10.1249/MSS.0000000000002712 
47. Vajro P, Lenta S, Socha P et al (2012) Diagnosis of nonalcoholic fatty liver disease in children and adolescents: position paper of the ESPGHAN Hepatology Committee. J Pediatr Gastroenterol Nutr 54:700-713. https://doi.org/10.1097/MPG.0b013e318252a13f

48. Labayen I, Ruiz JR, Ortega FB et al (2015) Liver enzymes and clustering cardiometabolic risk factors in European adolescents: the HELENA study. Pediatr Obes 10:361-370. https://doi.org/10.1111/ijpo.273

49. Borghese MM, Tremblay MS, LeBlanc AG et al (2017) Comparison of ActiGraph GT3X + and Actical accelerometer data in 9-11-year-old Canadian children. J Sports Sci 35:517-524. https://doi.org/10.1080/02640414.2016.1175653

50. Migueles JH, Cadenas-Sanchez C, Tudor-Locke C et al (2019) Comparability of published cut-points for the assessment of physical activity: Implications for data harmonization. Scand J Med Sci Sports 29:566-574. https://doi.org/10.1111/sms.13356

51. Fridolfsson J, Buck C, Hunsberger M et al (2021) High-intensity activity is more strongly associated with metabolic health in children compared to sedentary time: a cross-sectional study of the I.Family cohort. Int J Behav Nutr Phys Act 18:90. https://doi.org/10.1186/s12966-021-01156-1

\section{Tables}

Table 1: Anthropometric, accelerometry and hepatic variables for overall sample, SED-, SED+, PA+ and PA- groups. Model 1: univariate analysis. Model 2: adjusted with age, gender and Tanner. Model 3: adjusted with age, gender, Tanner and BMI. 


\begin{tabular}{|c|c|c|c|c|c|c|c|c|c|c|c|}
\hline & $\begin{array}{l}\text { Overall } \\
(n=134)\end{array}$ & $\begin{array}{l}\text { SED- } \\
(n=67)\end{array}$ & $\begin{array}{l}\text { SED+ } \\
(n=67)\end{array}$ & $\begin{array}{l}\text { p- } \\
\text { value } \\
\text { Model } \\
1\end{array}$ & $\begin{array}{l}\text { p- } \\
\text { value } \\
\text { Model } \\
2\end{array}$ & $\begin{array}{l}\text { p- } \\
\text { value } \\
\text { Model } \\
3\end{array}$ & $\begin{array}{l}\text { PA+ } \\
(n=67)\end{array}$ & $\begin{array}{l}\text { PA- } \\
(n=67)\end{array}$ & $\begin{array}{l}\text { p- } \\
\text { value } \\
\text { Model } \\
1\end{array}$ & $\begin{array}{l}\text { p- } \\
\text { value } \\
\text { Model } \\
2\end{array}$ & $\begin{array}{l}\begin{array}{l}\text { p- } \\
\text { value }\end{array} \\
\text { Model } \\
3\end{array}$ \\
\hline \multicolumn{12}{|l|}{ Anthropometry } \\
\hline Age (year) & $13.4 \pm 2.2$ & $13.1 \pm 2.3$ & $13.7 \pm 1.9$ & 0.093 & NA & NA & $13.0 \pm 2.3$ & $13.8 \pm 1.9$ & 0.024 & NA & NA \\
\hline Females $(\mathrm{n}, \%)$ & 65 (48.5) & $33(49)$ & $32(47)$ & 0.863 & NA & NA & $33(49)$ & $32(47)$ & 0.863 & NA & NA \\
\hline Tanner stage & $3.8 \pm 1.3$ & $3.7 \pm 1.3$ & $3.9 \pm 1.3$ & 0.549 & NA & NA & $3.7 \pm 1.3$ & $3.9 \pm 1.3$ & 0.747 & NA & NA \\
\hline $\mathrm{BMI}\left(\mathrm{kg} \times \mathrm{m}^{-2}\right)$ & $34.3 \pm 5.2$ & $32.9 \pm 5.5$ & $35.6 \pm 4.5$ & 0.002 & 0.017 & NA & $32.6 \pm 4.8$ & $36.1 \pm 5.0$ & $<0.001$ & 0.006 & NA \\
\hline $\begin{array}{l}\text { Z-BMI (Z- } \\
\text { score) }\end{array}$ & $3.18 \pm 0.48$ & $3.19 \pm 0.51$ & $3.16 \pm 0.45$ & 0.042 & 0.161 & NA & $3.14 \pm 0.48$ & $3.22 \pm 0.48$ & 0.041 & 0.189 & NA \\
\hline $\begin{array}{l}\text { BMI } \\
\text { (percentile) }\end{array}$ & $98.9 \pm 0.7$ & $98.8 \pm 0.8$ & $99.0 \pm 0.7$ & 0.015 & 0.014 & NA & $98.7 \pm 0.8$ & $99.0 \pm 0.7$ & 0.011 & 0.014 & NA \\
\hline WC (cm) & $109.0 \pm 13.8$ & $105.8 \pm 15.1$ & $112.3 \pm 11.5$ & 0.012 & 0.024 & 0.155 & $103.7 \pm 13.8$ & $114.3 \pm 11.7$ & $<0.001$ & 0.001 & 0.109 \\
\hline $\begin{array}{l}\text { FM percentage } \\
(\%)\end{array}$ & $44.0 \pm 7.3$ & $41.9 \pm 6.5$ & $46.1 \pm 7.6$ & 0.002 & 0.011 & 0.036 & $42.3 \pm 7.0$ & $45.7 \pm 7.3$ & 0.015 & 0.017 & 0.124 \\
\hline \multicolumn{12}{|l|}{ Accelerometry } \\
\hline $\begin{array}{l}\text { Sedentary } \\
\text { time } \\
\text { (min/day) }\end{array}$ & $640 \pm 116$ & $557 \pm 38$ & $723 \pm 109$ & $<0.001$ & $<0.001$ & $<0.001$ & $584 \pm 61$ & $696 \pm 171$ & $<0.001$ & $<0.001$ & $<0.001$ \\
\hline LPA (min/day) & $483 \pm 107$ & $533 \pm 88$ & $433 \pm 103$ & $<0.001$ & $<0.001$ & $<0.001$ & $494 \pm 80$ & $473 \pm 111$ & 0.401 & 0.276 & 0.322 \\
\hline $\begin{array}{l}\text { MPA } \\
\text { (min/day) }\end{array}$ & $186 \pm 76$ & $222 \pm 75$ & $149 \pm 59$ & $<0.001$ & $<0.001$ & $<0.001$ & $248 \pm 50$ & $124 \pm 84$ & 0.001 & $<0.001$ & $<0.001$ \\
\hline VPA (min/day) & $6 \pm 10$ & $8 \pm 14$ & $4 \pm 5$ & 0.023 & 0.047 & 0.145 & $10 \pm 13$ & $2 \pm 3$ & $<0.001$ & $<0.001$ & $<0.001$ \\
\hline $\begin{array}{l}\text { MVPA } \\
\text { (min/day) }\end{array}$ & $192 \pm 81$ & $231 \pm 81$ & $153 \pm 61$ & $<0.001$ & $<0.001$ & $<0.001$ & $258 \pm 56$ & $127 \pm 86$ & $<0.001$ & $<0.001$ & $<0.001$ \\
\hline $\begin{array}{l}\text { Total PA } \\
\text { (min/day) }\end{array}$ & $676 \pm 139$ & $764 \pm 72$ & $586 \pm 135$ & $<0.001$ & $<0.001$ & $<0.001$ & $752 \pm 81$ & $599 \pm 144$ & $<0.001$ & $<0.001$ & $<0.001$ \\
\hline \multicolumn{12}{|l|}{$\begin{array}{l}\text { Hepatic } \\
\text { variables }\end{array}$} \\
\hline ALT (U/L) & $32.8 \pm 27$ & $25.3 \pm 12$ & $40.3 \pm 34.9$ & 0.016 & 0.014 & 0.016 & $27.4 \pm 22.8$ & $38.1 \pm 29.8$ & 0.003 & 0.038 & 0.043 \\
\hline AST (U/L) & $28.9 \pm 12.7$ & $27.5 \pm 8.6$ & $30.4 \pm 15.9$ & 0.843 & 0.561 & 0.549 & $27.6 \pm 8.7$ & $30.2 \pm 15.7$ & 0.608 & 0.631 & 0.611 \\
\hline AST/ALT ratio & $1.07 \pm 0.39$ & $1.18 \pm 0.35$ & $0.96 \pm 0.05$ & $<0.001$ & 0.004 & 0.006 & $1.20 \pm 0.39$ & $0.94 \pm 0.35$ & $<0.001$ & 0.007 & 0.009 \\
\hline GGT(U/L) & $21.4 \pm 14.3$ & $18.2 \pm 8.5$ & $24.7 \pm 17.8$ & 0.019 & 0.023 & 0.055 & $19.1 \pm 12.3$ & $23.7 \pm 15.7$ & 0.049 & 0.298 & 0.363 \\
\hline FLI & $34.2 \pm 27.2$ & $28.3 \pm 26.0$ & $40.4 \pm 27.3$ & 0.004 & 0.131 & NA & $23.6 \pm 23.3$ & $44.8 \pm 26.9$ & $<0.001$ & 0.001 & NA \\
\hline MRI-LFC (\%) ${ }^{a}$ & $6.3 \pm 7.2$ & $4.2 \pm 2.4$ & $8.0 \pm 7.3$ & 0.012 & 0.010 & 0.013 & $4.0 \pm 2.4$ & $8.1 \pm 9.0$ & 0.054 & 0.077 & 0.102 \\
\hline $\begin{array}{l}\text { MRI-LFC } \geq 5 \% \\
(n, \%)^{a}\end{array}$ & $18(46)$ & $5(29.4)$ & $13(60.0)$ & 0.064 & 0.056 & 0.067 & $7(41.2)$ & $11(50.0)$ & 0.412 & 0.765 & 0.989 \\
\hline
\end{tabular}

ALT: alanine aminotransferase; AST: Aspartate aminotransferase; BMI: body mass index; FLI: Fatty Liver Index; FM: fat mass; GGT: gamma-glutamyl transferase; LFC : liver fat content; LPA: light physical activity; MPA: moderate physical activity; MRI: magnetic resonance imaging; MVPA: moderate to vigorous physical activity; NA . no-adjustment; VPA: vigorous physical activity; WC: waist circumference. ${ }^{a} n=39$ ( $n=17$ in SED-group, $n=22$ in SED+ group, $n=$ 17 in MVPA+ group and $n=22$ in MVPA-group)

Table 2: Anthropometric, accelerometry and hepatic variables for SED-/MVPA+, SED-/MVPA-, SED+/PA+ and SED+/MVPA-. P-values are adjusted with age, gender and Tanner. 


\begin{tabular}{|c|c|c|c|c|}
\hline & $\begin{array}{l}\text { SED-/MVPA+ } \\
(n=33)\end{array}$ & $\begin{array}{l}\text { SED-/MVPA- } \\
(n=34)\end{array}$ & $\begin{array}{l}\text { SED+/MVPA+ } \\
(n=33)\end{array}$ & $\begin{array}{l}\text { SED+/MVPA- } \\
(n=34)\end{array}$ \\
\hline \multicolumn{5}{|c|}{ Anthropometry and body composition } \\
\hline Age (year) & $12.4 \pm 2.3$ & $13.6 \pm 2.2$ & $13.1 \pm 1.8$ & $14.2 \pm 1.9$ \\
\hline Females (n, \%) & $15(45)$ & $17(50)$ & $16(48)$ & $18(52)$ \\
\hline Tanner stage & $3.4 \pm 1.5$ & $3.9 \pm 1.3$ & $3.7 \pm 1.3$ & $4.1 \pm 1.2$ \\
\hline $\mathrm{BMI}\left(\mathrm{kg} \times \mathrm{m}^{-2}\right)$ & $31.3 \pm 4.6$ & $34.6 \pm 5.9$ & $35.3 \pm 4.8$ & $36.0 \pm 4.3$ \\
\hline Z-BMI (z-score) & $3.24 \pm 0.54$ & $3.14 \pm 0.48$ & $3.18 \pm 0.48$ & $3.17 \pm 0.43$ \\
\hline BMI (percentile) & $98.7 \pm 0.8$ & $98.8 \pm 0.8$ & $98.9 \pm 0.7$ & $99.0 \pm 0.6 / a$ \\
\hline WC (cm) & $99.9 \pm 11.9$ & $111.6 \pm 15.9$ & $110.9 \pm 12.3$ & $113.6 \pm 10.8$ * \\
\hline FM percentage (\%) & $41.9 \pm 6.6$ & $42.0 \pm 6.5$ & $46.0 \pm 6.9 * \#$ & $46.1 \pm 7.5 * \#$ \\
\hline \multicolumn{5}{|l|}{ Accelerometry variables } \\
\hline Sedentary time (min/day) & $553 \pm 44$ & $562 \pm 31$ & $674 \pm 50 * * \# \# \# \#$ & $769 \pm 129$ **\#\#\#+† \\
\hline Light PA (min/day) & $496 \pm 82$ & $570 \pm 78$ & $460 \pm 59 \# \# \#$ & $408 \pm 127$ **\#\# \\
\hline Moderate PA (min/day) & $281 \pm 46$ & $165 \pm 49 * * *$ & $197 \pm 37$ **\#\# & $104 \pm 35 * * * \# \#+t+$ \\
\hline Vigorous PA (min/day) & $13 \pm 18$ & $4 \pm 5$ ** & $6 \pm 5$ & $2 \pm 2 * * *++$ \\
\hline MVPA (min/day) & $294 \pm 53$ & $169 \pm 51 * * *$ & $203 \pm 38$ **\#\#\# & $106 \pm 36$ ***\#\#+† \\
\hline Total PA (min/day) & $790 \pm 65$ & $739 \pm 71$ & $663 \pm 68$ * & $514 \pm 143$ ***\#\#"t+ \\
\hline \multicolumn{5}{|l|}{ Hepatic variables } \\
\hline $\operatorname{ALT}(\mathrm{U} / \mathrm{L})$ & $23.5 \pm 11.9$ & $26.9 \pm 12.0$ & $40.5 \pm 37.7$ *\# & $40.7 \pm 32.5$ **\# \\
\hline AST (U/L) & $27.3 \pm 7.5$ & $27.6 \pm 9.6$ & $29.7 \pm 10.8$ & $31.1 \pm 19.5$ \\
\hline AST/ALT ratio & $1.27 \pm 0.36$ & $1.10 \pm 0.32$ & $0.99 \pm 0.41$ *\#\# & $0.93 \pm 0.41$ *\#\# \\
\hline GGT (U/L) & $17.3 \pm 7.0$ & $18.9 \pm 9.7$ & $25.4 \pm 18.3$ *\# & $25.9 \pm 17.5$ *\# \\
\hline FLI & $18.5 \pm 19.3$ & $37.2 \pm 28.4$ & $38.8 \pm 29.2$ & $41.9 \pm 25.8$ \\
\hline MRI-LFC (\%) ${ }^{a}$ & $3.81 \pm 1.97$ & $4.47 \pm 2.25$ & $4.63 \pm 2.26$ & $11.24 \pm 10.78$ ** \\
\hline MRI-LFC $\geq 5 \%(n, \%)^{a}$ & $2(33.3)$ & $3(27.3)$ & $5(45.4)$ & $8(72.7)$ \\
\hline
\end{tabular}

ALT: alanine aminotransferase; AST: Aspartate aminotransferase; BMI: body mass index; FLI: Fatty Liver Index, FM: fat mass; GGT: gamma-glutamyl transferase; LFC : liver fat content; $L P A$ : light physical activity; MPA: moderate physical activity; MRI: magnetic resonance imaging; MVPA: moderate to vigorous physical activity; VPA: vigorous physical activity; WC: waist circumference

Different from SED-MVPA+:* $p<0.05 ; * * p<0.01 ; * * * 00.001$

Different from SED-MVPA- : $p<0.05 ; \# \# p<0.01$;\#\# $p<0.001$

Different from SED+MVPA+; $p<0.05 ;{ }^{\dagger+} p<0.01 ;+++; p<0.001$

a $n=39$ ( $n=11$ in SED-/MVPA+ group, $n=6$ in SED-/MVPA-group, $n=11$ in SED+/MVPA+ group and $n=11$ in SED+/MVPA- group)

\section{Figures}




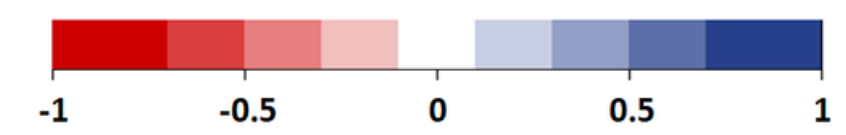

\begin{tabular}{|c|c|c|}
\hline$*$ & $* * *$ & $* * *$ \\
\hline$*$ & $* *$ & $*$ \\
\hline$*$ & $* *$ & * \\
\hline$*$ & $* *$ & $* *$ \\
\hline$* *$ & $* *$ & $* *$ \\
\hline$*$ & * & $*$ \\
\hline$* * *$ & $* * *$ & $* * *$ \\
\hline$*$ & $* * *$ & $* * *$ \\
\hline
\end{tabular}

BMI $\left(\mathrm{kg} . \mathrm{m}^{-2}\right)$
BMI-SDS
BMI-percentile
WC (cm)
FM (\%)
ALT (UI.L-1)
AST (UI.L-1)
AST/ALT ratio
GGT (UI.L-1)
FLI

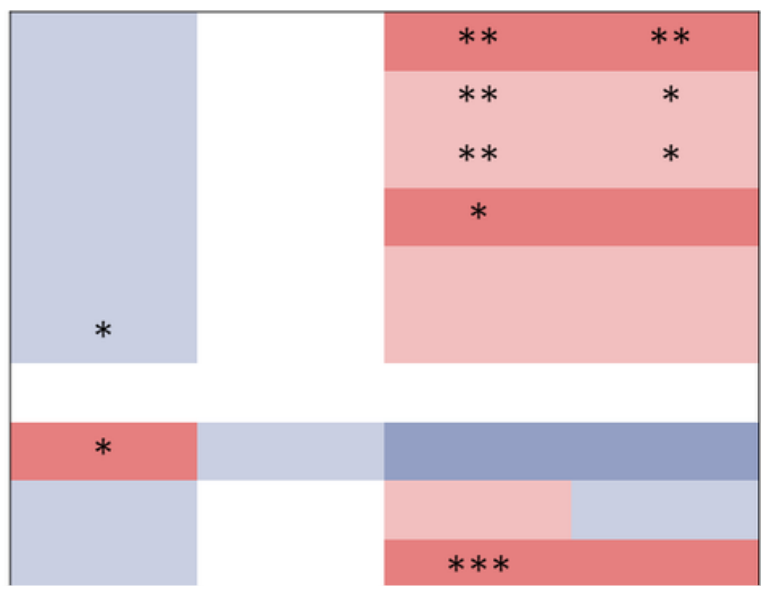

Figure 1

Heatmap representation of correlations between anthropometric, hepatic and accelerometry variables. The darkest is the box, the higher is the correlation. Left: unadjusted model. Right: adjusted for sedentary time when physical activity was modelled as the main exposure and for MVPA when sedentary time was modelled as the main exposure. ${ }^{*} p<0.05,{ }^{* *} p<0.01$, ${ }^{* *} p<0.001$

ALT: alanine aminotransferase; AST: aspartate aminotransferase; BMl: body mass index; FLI: fatty liver index; FM: fat mass; GGT: gamma-glutamyl transpeptidase; LFC: liver fat content; LPA: light physical activity; MRI: magnetic resonance imaging; MVPA: moderate to vigorous physical activity; PA: physical activity; SED: sedentary; WC: waist circumference

\section{Supplementary Files}

This is a list of supplementary files associated with this preprint. Click to download.

- Table1supplementaryfile.docx

- Table2supplementaryfile.docx 